\title{
Design of a Beat Frequency Oscillator Metal Detector
}

\author{
Aqeel Mahmood Jawad ${ }^{1}$, Haider Mahmood Jawad ${ }^{2}$, and Goh Chin Hock ${ }^{3}$ \\ ${ }^{1,2}$ Al Rafidain university college, Dept. of Electronic and Communication Engineering, Iraq \\ ${ }^{3}$ Universiti Tenaga Nasional, Dept. of Electronic and Communication Engineering, 43000 Kajang, Selangor, \\ Malaysia
}

\begin{abstract}
This paper discusses the design,construction, and simulation of a beat frequency oscillator metal detector. Over the years, it has become an important issue to limit, the entry of people into certain places with weapons or instruments of harm and destruction.in this paper is a design of an effective means of detecting dangerous weapons made of metal in order to reduce crime rate in the society. It uses the principle of electromagnetic induction and adopts a digital beat frequency technique. The system uses a search coil to detect metallic objects at a close range and gives a visual, auditory and vibratory indication once the metallic object is detected. The search coil forms part of an LC oscillation circuit. The activation frequency of the LC oscillator is primarily determined by the value of involved inductor and capacitor. In case an inductance is altered, the frequency does not remain the same by changing its value through the introduction of a metallic object.

Keywords: Metal;Detector; Oscillator; BFO; Frequency.
\end{abstract}

\section{INTRODUCTION}

The meaning of metal detectors consists often in demining, searching of Explosive Remnants of War (ERW), Unexploded Ordnance (UXO) or valuable metal objects by private persons [1,2]. It could be advantageous to have higher resolution capability in these spheres of action during the detection of metal objects. As it can be seen in some measured results using standard acoustic output and in [3], detectable measurable distance from detector center to rely on the size of tested object. These characteristics are situated in saturation for certain time and there is no possibility to extract required information about tested object from this output of the metal detector. The main task of this work is to increase resolution capability of metal detector. The simplest method is to find an appropriate usable output signal in detectors electronic. The original signal of the detector's search-head is processed in a few levels; according to[4] we should be able to discover some signals, whose parameters could be proportional to the size and distance of the metal object without the saturation and with more recognizable differences between single characteristics against the acoustic output. These signals could be more used for differentiation of searched objects from the others, which is useful for the convenience of work, for ex. Through demining, mapping of signal intensity or searching of specific valuable objects. On the other side some characteristic signals be able to suppressed in order to ignore uninteresting objects, which be able to increase work speed and efficiency [3]. Metal detectors work very well they are regarded a mature technology and can accurately detect the presence of most types of firearms and knives. though, metal detectors work very poorly if the user is not aware of their limitations before beginning a weapon detection program and is not prepared for the amount of trained and motivated manpower required to operate these devices successfully. A metal detection device in school security performance is used primarily to locate undesirable objects that are hidden on a person's body. When a confutable item or material is detected by the device, the detector produces an alarm signal; this signal can be audible, visible (lights), or both. Unluckily, a metal detector alone cannot distinguish between a gun and a large metal belt buckle. This imperfection is what makes weapon detection programs impractical for many schools; trained employees are needed to make these determinations [4]. A metal detector is only as good as the operator overseeing its use. In many facilities, the misconception exists that someone familiar by the operator, like a fellow employee or a security person, should be allowed to circumvent the system. It must be apparently established that in order to ensure the integrity of any routine metal detection program, everyone must be subjected to the program requirements, including students, parents, teachers, custodial and maintenance staff, security personnel (except for sworn police officers who are required to carry a weapon), school administrators, and visitors. To require less would be counterproductive and prejudicial. Sign age can be of large help: a sign at the school entrance explaining the importance of the detectors in maintaining a safe and comfortable learning environment provides policy notification. If a more aggressive mehod is needed for a particular community, entry signs could spell out a particular school or district policy that requires the screening of all who enter the school, with access denied to those who refuse [4].Metal detectors are usually not effective when used on purses, book bags, briefcases, or suitcases. There is usually a large number of different objects or materials located in or as part of the composition of these carried items that would cause an alarm. If you ask the average person what a metal detector does and what property to which it is most sensitive, the answer to the first question would probably be 
that it is a device that detects only metal. The answer to the second question likely would be that a metal detector is more likely to detect metal objects with heavier mass. Both answers are incorrect. A metal detector actually detects any conductive materialanything that will conduct an electrical current. The stereotyped pulsedfield portal metal detectors generate electromagnetic pulses that produce very small electrical currents in conductive metal objects within the portal archway which, in turn, generate their own magnetic field.The receiver portion of a portal metal detector can detect this rapidly decaying magnetic field within the time between the transmitted pulses. This kind of weapon detection device is "active" in that it generates a magnetic field that energetically looks for suspicious materials or objects [5]. A magnetometer, a passive device, was much greater in use 20 years ago in the detection of weapons. The magnetometer to rely on the Earth's magnetic field-it looks for a distortion caused by the attendance of ferromagnetic (attracted to a magnet) material. Counter to intuition, the mass of a specific, object is not significant in metal detection. The size, shape, electrical conductivity and magnetic properties are the significant properties. For example, when a long thin wire is taken through a portal (walk-through) metal detector, and the wire is in any geometry except one in which the two ends (or any two points on the wire) are touching, it will not often be detected. However, shape this same wire into a closed circle and the metal detector will probably go off, even though the mass of the wire has not changed. Delving even deeper into metal detector sensitivity, regard as the orientation of an object [6]. Take the same closed-loop wire to depict in the prior paragraph. Lay this loop on its side so that it is parallel to the ground. In this shape, the portal metal detector is less likely to see it, but, if the wire loop is upright and parallel to the side panels of the metal detector, the detector will be much more probable to go off in this orientation. Some people fear the use of a metal detector on themselves because of the can be achieved side effects of being subjected to the magnetic field. This fear is baseless; metal detectors emit an exceedingly weak magnetic field, weak sufficient to be of no concern even to heart patients with pacemaker-type devices. Indeed, the use of an electric hair dryer subjects the user to a much stronger field than assuming received by a metal detection device. Another greatly held belief about metal detectors is that they are a straightforward technology, where the equipment does all the work. This is not true at all. The average first-time consumer will certainly expect a metal detector to be much smarter and greater contributory, than it can maybe [7].

\section{THE METAL DETECTOR THEORY}

The search-head of the detector is equipped with two coils. If an electric current is passed through the first coil, the primary magnetic field is generated. This field causes an origin of the secondary magnetic field around a metal object, which is located under the detector searchhead. The secondary field changes in time along with the primary field and it's detected by the second coil in the search-head where an electric voltage is induced according to Faraday's law of induction [8].

$\varepsilon=-\frac{d \phi}{d t}$

This law says, the induced electromotive force $\varepsilon$ in any closed circuit is equal to the time rate of change of the magnetic flux $\phi$ through the circuit. A second expression of the Faraday's law is through the use of Maxwell's equation [8]:

$\oint_{C} E . d l=-\frac{d \phi}{d t}=-\frac{d}{d t} \oint_{S} B . d s$

This equation denote, that the line integral of the electric field intensity $E$ along the boundary $C$ of a surface $S$ equates to the magnetic flux through the surface $S$ ( $B$ means magnetic induction). The voltage created by the secondary magnetic field is further rectified and converted to the frequency, which is proportional to the size of original voltage. This is only simplified popular, there are some negative factors too, that are solved in electronic circuits.

\section{BFO DETECTOR}

The beat-frequency oscillator, or BFO, is generally the simplest type of metal detector and is a good starting point for learning how metal detectors work. The dominant technology throughout the 60's and much of the 70's, the BFO has now practically vanished from the market and can only be found in the poorest quality toy detectors. Although it has run its course commercially, the BFO can still make a pretty good detector and there are a few tricks that can make it an excellent detector under some conditions. We will not investigate these "tricks" in this paper but will focus on the fundamentals. originally, it seems the beat frequency oscillator detector will be the best choice to build caused by its relatively simple circuitry, which will allow us to focus more on the electromagnetinc theory during the project [9]. The basic way the Beat Frequency Oscillator (Later only BFO) works, when the detector coil is above some metal, it will change the frequency in the detector oscillator, which has the detector coil in the frequency depended circuit. The detected frequency is compared to 
a reference oscillator in a mixer, so there will be both the different and the sum of the 2 frequencies. The detector we has made isn't really a real BFO, while the reference is internal in a Micro Controller (Later only $\mu \mathrm{C}$ ) and the signal from the detector oscillator is connected directly to the $\mu \mathrm{C}$ 's external timer pin. In the code for the $\mu \mathrm{C}$ there is implemented an average function, so if the ground has high magnetic fields it will compensate for it after some seconds. The output is indicated by Light Emitted Diodes (Later only LED) and by a sound in different locked frequencies. When some metal is moving close to a coil the magnetic field around the coil is changed and the coil inducts some energy, called Eddie current. The same principle is true, if there is putted some energy in the coil it changes the magnetic field around the Coil. The way is also used in loudspeakers, when it is playing, the energy is conducted to the speaker, and if there is measured on the speaker and pushes a little to the membrane, the speaker generates some energy. If the terminals on the speaker are shorted, the membrane is hard to push, the coil can't make the energy, and the coil is locked. But the difference between the speakers and the earth is that the speakers have big magnetic part to help the membrane to move, otherwise in the metal detector it is normally not a magnetic object there has to be detected, so the coil has to produce it own magnetic field. When the detectors magnetic beams are reaching a metal, the metal start to induct the fields, and reply the magnetic field in another direction / time, this change can be seen in the frequency / pulse response of the coil. There are big different there are 2 major groups of detectors: Passive detector uses the detector coil in a frequency depended part of a circuit, example a Oscillator where the inductance of the coil and the capacitive of a capacitor are making a oscillation, when these parts have positive feedback, and the amplifier a gain of 1 , it will continue oscillating. When some metal is coming close to the alternating magnetic field, the metal changing the field, and the inductance in the coil changes a little, and then the frequency. Active detectors uses the coil to transmit a pulse or a continually waveform, some uses the same coil to receive with, and others have 1 or 2 receiving coils [10]. The PI loads the coil with some current in a narrow pulse, and when it releases the coil it make a reflective pulse the duration of the reflected pulse is only a few $\mu \mathrm{S}$, and the pulse can be several 100v high [11]. When some metal are coming close to the coil the amplitude of the reflective pulse is getting little lower and the duration of the pulse a little longer, almost like the metal behaves like a capacitor for magnetic energy, in the top of the reflective the metal collect magnetic energy, and when the pulse is falling in voltage it returns the energy slowly. Different metal, have different reaction time. The sampled signal has to be amplified up to a signal that can be used. Expensive metal detectors can show which kind of metal there is registered, and even be setup to discriminate between them, so there wont be any kind of detection it comes near an old can, but if it is gold or other nonferrous metal it shows some result. The way that can be done is to closely analyse the returned signal. VLF principle is the best type to discriminate metal, it sends out a constant magnetic field, and receive it in another coil. When the received field is changed in the phase there must be a metal close. The amplitude of the signal tells how deep / big the object is [12].

\section{BFO DETECTOR CIRCUIT DESIGN}

This related, simple metal detector isn't a real BFO, but it is the near group of detectors. As shown in Figure 1 the Oscillator makes a frequency change when some metal is near the detector coil. The signal is send to a timer pin on the $\mu \mathrm{C}$. Internal in the $\mu \mathrm{C}$ the calculation is made, and showed on the LED and some different sounds are made. The $\mu \mathrm{C}$ gives a big advantage of detecting the signal it makes detection of small changes down to a few hertz. Average is also a function the $\mu \mathrm{C}$ can manage, and then it can discriminate high level of magnetic fields in the earth, a kind of auto gain control [13-15].

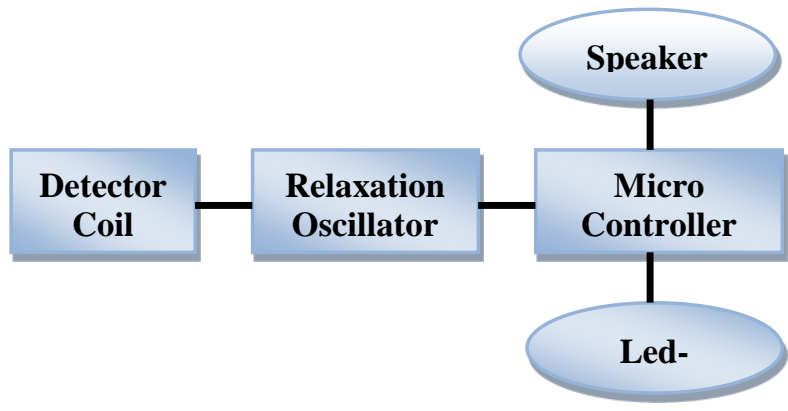

Figure 1

\section{A. Coil \& Oscillator Design}

The coil is a flat spiral coil, with 13 turns around an old CD, which have the diameter of $12 \mathrm{~cm}$. The used copper wire has a diameter of $1.5 \mathrm{~mm}$. The coil is made in the spiral shape to get the magnetic field as wide as 
possible. Different types of coils where tested, but this seemed to be the best. The metal sensing part of our designed metal detector, is built around an inverter, where the frequency depended components is the detector coil and the 2 capacitors. The inverter delivery enough power to the oscillation parts, so it continues to oscillate. The oscillator always starts when the power is turned on, while the dc level on the output is inverted and dc coupled to the input. The LC components limit the frequency to the oscillating frequency. The oscillating frequency can approximately be calculated using this formula [16]:

$F_{O S C}=\frac{1}{2 \pi \sqrt{L * C}}=175.4 \mathrm{kHz}$

The 2 capacitors are coupled in series, through the ground, and it makes a parallel oscillation circuit. The resistor in the top gives the coil a little lower Q, and the oscillation a higher bandwidth. The Calculation of coil in the Relaxation oscillator can be determined from below equations[16].

Coil Wire Length :

$P i *($ Diameter $+($ Coil Wire Diameter $*$ Turns on coil $=5.71 \mathrm{~m}$

Dc Resistance in Coil:

$R_{\text {wire }}=\frac{\left(R_{\text {material }} \text { Length }\right)}{\text { Cross Section Area }}=0.056518 \Omega$

AC characteristics for the coil :

Average Radius :

$A=\frac{\frac{D i}{25.4}+N\left(\frac{W}{25.4}+\frac{S}{25.4}\right)}{2}=2.746$ Inch

Coil inductance :

$\mathrm{L}=\frac{\mathrm{N}^{2} * \mathrm{~A}^{2}}{30 * \mathrm{~A}-11 *\left(\frac{\mathrm{Di}}{25.4}\right)}=41.91 \mu \mathrm{H}$

Where:

$\mathrm{N}=$ Turns on coil;

A=Average Radius;

Di=Inner Diameter;

$\mathrm{W}=$ Wire width;

$\mathrm{S}=$ Spacing between wires

$\mathrm{C}_{\text {Total }}=\frac{39 \mathrm{nF} * 39 \mathrm{nF}}{39 \mathrm{nF}+39 \mathrm{nF}}=19.5 \mathrm{nF}$

$\mathrm{Q}_{\text {Coil }}=\frac{\sqrt{\frac{\mathrm{L}}{\mathrm{C}}}}{\mathrm{R}_{\text {wire }}}=816 \mathrm{Q}$

$Z_{\text {resonans }}=R_{\text {wire }}\left(1+Q_{\text {Coil }}^{2}\right)=38 \mathrm{~K} \Omega$

$Q_{N e w}=\sqrt{\frac{Z_{\text {Total }}}{R_{\text {wire }}}}=813 \mathrm{Q}$

\section{B. Microcontroller $(\mu C)$ Circuit Design}

Microcontroller $(\mu \mathrm{C})$ Circuit Design as shown in Figure 2 the function of the $\mu \mathrm{C}$ is to compare the input frequency with a reference, in this case an internal timer in the $\mu \mathrm{C}$, it counts for approximate $250 \mathrm{mS}=4$ samples in a second. The frequency from the oscillator is connected to an internal 16bit counter, every time the timer generates an overflow the counter is read. The result of the counter $* 4$ gives the incoming frequency, but there is no need to now the frequency, only the difference is important. When the detector is turned on, the 
oscillator has to stabilize, the $\mu \mathrm{C}$ waits until the frequency is stable, in the meanwhile all LED's are flashing. The detector is averaging the incoming signal all the time, if the same frequency has been present for 16 measurements the frequency will be the new zero point. It is necessary for using the detector in areas with high level of iron in the ground. The speaker and LED indicates the level of detected material, in zero point it is around the middle of the area, so it can detect both ferrous and nonferrous metal, and make different output of it. The $\mu \mathrm{C}$ reset circuit is $\mathrm{R} 2 \& \mathrm{C} 12$, which makes a time delay before the reset pin is on the high level, the $\mu \mathrm{C}$ doesn't start before the reset pin is high. The capacitor on the loudspeaker is the ensure there only flows a current in the changes of the signal [16].

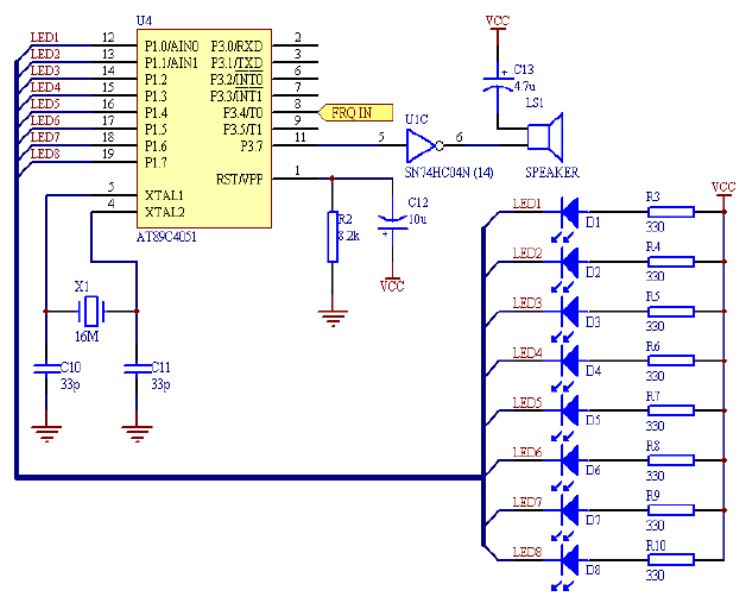

Figure 2

\section{SIMULATION BFO DETECTOR CIRCUIT DESIGN}

The simulation is done by Proteus 8 Professional software.The following simulation are made with a $15 \mathrm{Kr}$ coin in different positions from the coil search the simulations are the result in Table 1.In Figure 3 shown the relation between distance from the coli search and the loudspeaker frequency, also the Figure 4 describe the relation between distance from the coli search and oscillator frequency .

Table 1

\begin{tabular}{|c|c|c|}
\hline Distance from the Coil $[\mathrm{cm}]$ & $\begin{array}{c}\text { Oscillator } \\
\text { Frequency } \\
{[\mathrm{kHz}]}\end{array}$ & Spk. Frequency [Hz]: \\
\hline 0 & 180.40 & 15000 \\
\hline 0.5 & 180.28 & 14800 \\
\hline 1 & 180.12 & 13500 \\
\hline 1.5 & 180 & 12000 \\
\hline 2 & 178.2 & 11100 \\
\hline 2.5 & 177.4 & 10200 \\
\hline 3 & 177.4 & 9700 \\
\hline 3.5 & 177.4 & 9200 \\
\hline 4 & 177.4 & 8000 \\
\hline 4.5 & 177.4 & 7600 \\
\hline 5 & 177.4 & 7400 \\
\hline 5.5 & 177.3 & 7100 \\
\hline 6 & 176.2 & 6800 \\
\hline 6.5 & 176.2 & 6200 \\
\hline 7 & 176.2 & 6000 \\
\hline 7.5 & 176.2 & 5700 \\
\hline 8 & 176.2 & 5400 \\
\hline 8.5 & 175.8 & 5200 \\
\hline 9 & 175.8 & 4000 \\
\hline 9.5 & 175.8 & 3750 \\
\hline 10 & 175.8 & 3500 \\
\hline 10.5 & 175.8 & 3150 \\
\hline 11 & 175.8 & 3050 \\
\hline 11.5 & 175.8 & 2900 \\
\hline 12 & 175.8 & 2700 \\
\hline 12.5 & 175.5 & 2650 \\
\hline 13 & 175.5 & 2400 \\
\hline 13.5 & 175.5 & 2200 \\
\hline
\end{tabular}




\begin{tabular}{|c|c|c|}
\hline 14 & 175.5 & 2100 \\
\hline 14.5 & 175.5 & 2050 \\
\hline 15 & 175.5 & 1900 \\
\hline 15.5 & 175.5 & 1820 \\
\hline 16 & 175.5 & 1730 \\
\hline 16.5 & 175.5 & 1675 \\
\hline 17 & 175.25 & 1500 \\
\hline 17.5 & 175.25 & 1440 \\
\hline 18 & 175.25 & 1400 \\
\hline 18.5 & 175 & 1100 \\
\hline 19 & 175 & 1000 \\
\hline 19.5 & 175 & 750 \\
\hline 20 & 175 & 0 \\
\hline
\end{tabular}

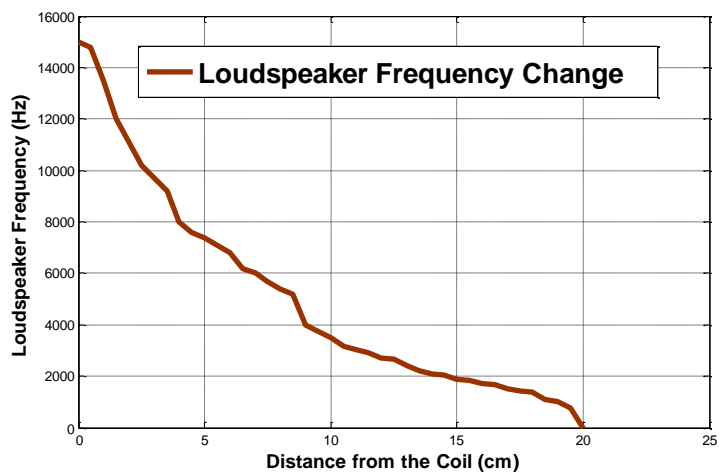

Figure 3

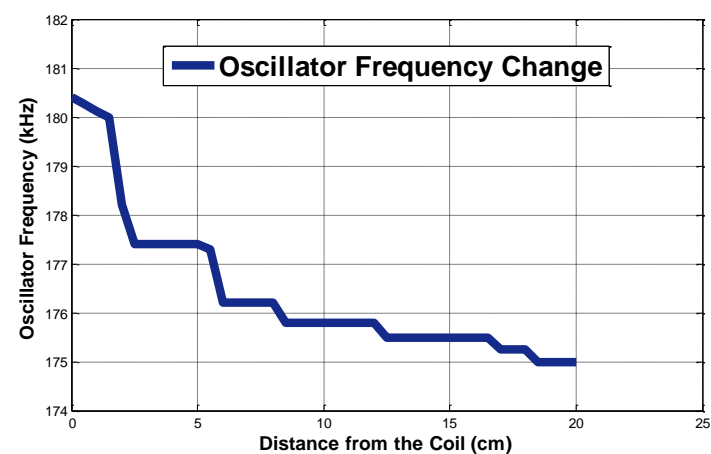

Figure 4 .

\section{ANALYSIS}

Like all metal detectors, it uses metal coils to help discern both the proximity of the metal as well as the type of metal with enough research. How this works is by running a current through these coils. When the coil is charged, the shape of the charged coil creates a magnetic field that flows through the center of the coils, out around then back into them again. This electromagnetic field is what reacts to nearby metals. When the field comes in contact with another metal, that metal then creates its own electromagnetic field that flows opposite to the field created by the coils. That opposing field in turn decreases the inductance of the original metal coils. All of the different kinds of metal detectors process and measure this change in some way, but we will concentrate on how that is specifically done in a beat frequency oscillator. The beat frequency oscillator metal detector essentially creates a coil with an inductance within the circuit that is kept constant. The inductance of the coil next to the metal is compared to that of the internal coil. The difference is then translated into an audio output that changes relative to the size of the difference in inductances. The coil with an inductance held constant is called the reference coil, while the coil that detects metal is called the search coil. The detector depends on the difference between the inductances of the two coils to function correctly; so when there are no metals nearby, the inductance of the two coils should be equal to each other. Of course this probably will not hold true when the circuit is first built. Every measurement tool needs calibration of some sort when it is first built. To do so in this particular circuit, we must use the P1 potentiometer. It is there to alter the pulses going into the coil and thus alter the inductance. Calibrating is a matter of changing the resistance until the reference coil's inductance is equal to that of the search coil when there are not any metals close by. Empirically, it is almost impossible for 
both coils to have identical inductances, but it can be close enough so that any change in inductance is readily noticed through the audio output. the circuit.

Though it is the inductance that is directly affected by nearby metals, it is not directly measured within

\section{CONCLUSIONS}

Our original design aims were to be able to detect a small piece of metal, such as a coin, buried under approximately two inches of dirt. Unfortunately, after construction and initial testing, it was found that our metal detector was simply not that sensitive. In fact, it took a noticeably large amount of metal (on the order of 1-2 kg) in order to change the search coil's inductance enough to produce a noticeable beat. There seems to be a lot of issues to consider in a BFO design, but compared to the induction-balance detector it is very simple. The simplicity of the coil makes it easy to try out different shapes, sizes, and windings to compare sensitivities. The oscillator frequencies can be varied for even more comparisons. Such information will often carry over to more advanced designs. This makes the BFO an excellent first project that provides a solid foundation for learning.

\section{REFERENCES}

[1] Carin, L.: (2001), Special Issue on Landmine and UXO Detection, IEEE Transactions on Geoscience and Remote Sensing, Vol. 39 No 6.

[2] Daniels, D.;(2001), Cespedes E.: Special Issue UXO and Mine Detection, Subsurface Sensing Technologies and Applications (SSTA), Vol. 2 Issue 3.

[3] Aubrey Lee Kozak, (2003), Underwater Metal Detection Using Video Image Processing Techniques to Determine Location, Thesis. Available from World Wide Web http://www.giscogeo.com/pages/giscomtl.html

[4] SEIGENFELD, A.. ATMID - TECHNOLOGIE - und SCHALTUNGSBESCHREIBUNG.(2003). $55 \mathrm{~s}$.

[5] MACDONALD, J., et al. Alternatives for landmine detection. [s.1.] RAND, (2004). 336s. Available from World Wide Webhttp://demining.jrc.it/aris/events/mine99/program/P155-160/MINE99PO.htm.

[6] J. D. Kraus and D. A. Fleisch. Electromagnetics With Applications. Mc-Graw Hill, 5nd edition,(1999). ISBN 0-07-289969.

[7] Szyngiera, P.: A Method of Metal Object Identification by Electromagnetic Means, in Proc.MINE'99 (Mine Identification Novelties Euroconference), Florence, Italy, pp. 155-160 (1999). Available from World Wide Web http://demining.jrc.it/aris/events/mine99/index.htm.

[8] GUELLE, Dieter, et al. Metal detector handbook for humanitarian demining.Luxembourg: Office for Official Publications of the European Communities, 2003. 172 s. ISBN 92-894-6236-1. Available from World Wide Webhttp://www.nuggetshooter.com/articles/UnderstandingPIdetector.html.

[9] RIGGS, L., et al. Discrimination experiments with the U.S. Army's standard metal detector. Radio Science. 2004, vol. 39, no. 4, RS4001 10.1029/2003RS002994.

[10] S. Yamazaki, H. Nakane, and A. Tanaka. (2001), Basic analysis of a metal detector. IEEE Instrumetation and Measurement.

[11] T. A.-. P. G. 08gr1041, AAU (2008). 'New Generation Metal Detector for Food' by Frank Thornemann Hansen \& Lars Vinding, Graduate Master Students, 08gr1041@es.aau.dk

[12] Pavel Blažek "INTELLIGENT METAL DETECTOR", CZECH TECHNICAL UNIVERSITY IN PRAGUE,FACULTY OF ELECTRICAL ENGINEERING, DEPARTMENT OF MEASUREMENT, THESIS, (2010). Available from World Wide Webhttp://www.deepfriedneon.com/tesla f calcspiral.html 